\title{
On Functional Relations for Witten Multiple Zeta-functions
}

\author{
Soichi IKEDA and Kaneaki MATSUOKA \\ Nagoya University and Nagoya University \\ (Communicated by M. Tsuzuki)
}

\begin{abstract}
In this paper we discuss functional relations for the multi-variable version of Witten zeta-functions associated with the Lie algebra $\mathfrak{s l}(r)(r=3,4,5)$.
\end{abstract}

\section{Introduction}

Let $\mathfrak{g}$ be a semisimple Lie algebra and $s \in \mathbf{C}$. The Witten zeta-function $\zeta_{\mathfrak{g}}(s)$ attached to $\mathfrak{g}$ is defined by

$$
\zeta_{\mathfrak{g}}(s)=\sum_{\rho}(\operatorname{dim} \rho)^{-s}
$$

where $\rho$ runs over all finite dimensional irreducible representations of $\mathfrak{g}$. Zagier defined the function $\zeta_{\mathfrak{g}}(s)$ in [12]. The special values of $\zeta_{\mathfrak{g}}(s)$ were introduced and studied by Witten in [11]. In [5] Matsumoto and Tsumura defined the multi-variable version of Witten zetafunctions associated with semisimple Lie algebra $\mathfrak{s l}(r+1)(r=1,2, \ldots)$

$$
\zeta_{\mathfrak{s l}(r+1)}(\mathbf{s})=\sum_{n_{1}, n_{2}, \ldots, n_{r}=1}^{\infty} \prod_{j=1}^{r} \prod_{k=1}^{r-j+1}\left(\sum_{v=k}^{j+k-1} n_{v}\right)^{-s_{j, k}}
$$

where

$$
\mathbf{s}=\left(s_{j, k}\right)_{1 \leq j \leq r, 1 \leq k \leq r-j+1} \in \mathbf{C}^{r(r+1) / 2} \quad\left(\Re s_{j, k}>1\right) .
$$

The general definition of the multi-variable version of Witten zeta-functions was given in [4]. Recently, the multi-variable version of Witten zeta-functions are also called the zeta-functions of root systems (see [3]). Since the values of the Witten zeta-function have applications for theoretical physics (see [11] and [12]), we may expect that the multi-variable version of Witten zeta-functions also have some applications. However, as far as the authors know, such applications have not been found yet.

Received August 19, 2014; revised July 2, 2015 
Some relations for the special values of certain multiple zeta-functions were studied by many authors, for example [2, 8, 9]. In [10] Tsumura found the functional relations between $\zeta_{\mathfrak{s l}(3)}$ and the Riemann zeta-function

$$
\begin{aligned}
& \zeta_{\mathfrak{s l}(3)}(k, l, s)+(-1)^{l} \zeta_{\mathfrak{s l}(3)}(s, l, k)+(-1)^{k} \zeta_{\mathfrak{s l}(3)}(s, k, l) \\
& =2 \sum_{\substack{j=0 \\
j \equiv k}}^{k}\left(2^{1-k+j}-1\right) \zeta(k-j) \\
& \quad \times \sum_{\mu=0}^{[j / 2]} \frac{(i \pi)^{2 \mu}}{(2 \mu) !}\left(\begin{array}{c}
l+j-2 \mu-1 \\
j-2 \mu
\end{array}\right) \zeta(s+l+j-2 \mu) \\
& -4 \quad \sum_{j=0}^{k}\left(2^{1-k+j}-1\right) \zeta(k-j) \sum_{\mu=0}^{[(j-1) / 2]} \frac{(i \pi)^{2 \mu}}{(2 \mu+1) !} \sum_{\substack{\nu=0 \\
(\bmod 2)}}^{l} \zeta(l-v) \\
& \quad \times\left(\begin{array}{c}
\nu+j-2 \mu-1 \\
j-2 \mu-1
\end{array}\right) \zeta(s+v+j-2 \mu) .
\end{aligned}
$$

In [6] Nakamura obtained

$$
\begin{aligned}
& \zeta_{\mathfrak{s l}(3)}(k, l, s)+(-1)^{l} \zeta_{\mathfrak{s l}(3)}(s, l, k)+(-1)^{k} \zeta_{\mathfrak{s l}(3)}(s, k, l) \\
& =2 \sum_{j=1}^{[k / 2]}\left(\begin{array}{c}
k+l-2 j-1 \\
k-2 j
\end{array}\right) \zeta(2 j) \zeta(s+k+l-2 j) \\
& +2 \sum_{j=1}^{[l / 2]}\left(\begin{array}{c}
k+l-2 j-1 \\
l-2 j
\end{array}\right) \zeta(2 j) \zeta(s+k+l-2 j) \\
& -\left(\left(\begin{array}{c}
k+l-1 \\
k
\end{array}\right)+\left(\begin{array}{c}
k+l-1 \\
l
\end{array}\right)\right) \zeta(s+k+l)
\end{aligned}
$$


by a method different from that of Tsumura. In [5] Matsumoto and Tsumura obtained

$$
\begin{gathered}
\zeta_{\mathfrak{s l}(4)}\left(s_{1}, 0, s_{2}, 2 k+1, s_{3}, 2 l+q\right)-(-1)^{q} \zeta_{\mathfrak{s l}(4)}\left(s_{1}, 0, s_{2}, 2 l+q, s_{3}, 2 k+1\right) \\
-\zeta_{\mathfrak{s l}(4)}\left(2 k+1, s_{2}, s_{1}, 2 l+q, 0, s_{3}\right)-\zeta_{\mathfrak{s l}(4)}\left(s_{1}, 2 l+q, 2 k+1, s_{2}, 0, s_{3}\right) \\
=\zeta_{\mathfrak{s l}(3)}\left(s_{1}+2 k+1, s_{2}+2 l+q, s_{3}\right) \\
-2 \sum_{j=0}^{k} \phi(2 k-2 j) \sum_{\rho=0}^{j}\left(\begin{array}{c}
2 l+q+2 j-2 \rho \\
2 j+1-2 \rho
\end{array}\right) \frac{(-1)^{\rho} \pi^{2 \rho}}{(2 \rho) !} \\
\times \zeta_{\mathfrak{s l}(3)}\left(s_{1}, s_{2}, s_{3}+2 l+2 j+q+1-2 \rho\right) \\
+4 \sum_{\nu=1}^{l} \zeta(2 l-2 v) \sum_{j=0}^{k} \phi(2 k-2 j) \sum_{\rho=0}^{j}\left(\begin{array}{c}
2 v+q+2 j-2 \rho \\
2 j-2 \rho
\end{array}\right) \frac{(-1)^{\rho} \pi^{2 \rho}}{(2 \rho+1) !} \\
\times \zeta_{\mathfrak{s l}(3)}\left(s_{1}, s_{2}, s_{3}+2 v+2 j+q+1-2 \rho\right)
\end{gathered}
$$

and

$$
\begin{aligned}
& \zeta_{\mathfrak{s l}(4)}\left(s_{1}, 0, s_{2}, 2 k, s_{3}, 2 l+q\right)+(-1)^{q} \zeta_{\mathfrak{s l}(4)}\left(s_{1}, 0, s_{2}, 2 l+q, s_{3}, 2 k\right) \\
& +\zeta_{\mathfrak{s l}(4)}\left(2 k, s_{2}, s_{1}, 2 l+q, 0, s_{3}\right)-\zeta_{\mathfrak{s l}(4)}\left(s_{1}, 2 l+q, 2 k, s_{2}, 0, s_{3}\right) \\
& =-\zeta_{\mathfrak{s l}(3)}\left(s_{1}+2 k, s_{2}+2 l+q, s_{3}\right) \\
& +2 \sum_{j=0}^{k} \phi(2 k-2 j) \sum_{\rho=0}^{j}\left(\begin{array}{c}
2 l+q+2 j-2 \rho-1 \\
2 j-2 \rho
\end{array}\right) \frac{(-1)^{\rho} \pi^{2 \rho}}{(2 \rho) !} \\
& \times \zeta_{\mathfrak{s l}(3)}\left(s_{1}, s_{2}, s_{3}+2 l+2 j+q-2 \rho\right) \\
& -4 \sum_{\nu=1}^{l} \zeta(2 l-2 v) \sum_{j=1}^{k} \phi(2 k-2 j) \sum_{\rho=0}^{j-1}\left(\begin{array}{c}
2 v+q+2 j-2 \rho-1 \\
2 j-2 \rho-1
\end{array}\right) \frac{(-1)^{\rho} \pi^{2 \rho}}{(2 \rho+1) !} \\
& \times \zeta_{\mathfrak{s l}(3)}\left(s_{1}, s_{2}, s_{3}+2 v+2 j+q+1-2 \rho\right)
\end{aligned}
$$

where $\phi(s)=\left(2^{1-s}-1\right) \zeta(s)$,

$$
C_{-q}\left(2 k+1 ; s_{1}, s_{2}, s_{3}\right)= \begin{cases}\zeta_{\mathfrak{s l}(3)}\left(s_{1}, s_{2}, s_{3}+2 k+1\right) & (\text { if } q=0) ; \\ \sum_{j=0}^{k} \phi(2 k-2 j) \sum_{\rho=0}^{j}(2 j-2 \rho+1) \frac{(-1)^{\rho} \pi^{2 \rho}}{(2 \rho+1) !} & \\ \times \zeta_{\mathfrak{s l}(3)}\left(s_{1}, s_{2}, s_{3}+2 j+2-2 \rho\right) & (\text { if } q=1)\end{cases}
$$


and

$$
C_{-q}^{*}\left(2 k ; s_{1}, s_{2}, s_{3}\right)=\left\{\begin{array}{cc}
-\zeta_{\mathfrak{s l}(3)}\left(s_{1}, s_{2}, s_{3}+2 k\right) & (\text { if } q=0) ; \\
\sum_{j=1}^{k} \phi(2 k-2 j) \sum_{\rho=0}^{j-1}(2 j-2 \rho) \frac{(-1) \rho^{2 \rho}}{(2 \rho+1) !} & \\
\times \zeta_{\mathfrak{s l}(3)}\left(s_{1}, s_{2}, s_{3}+2 j+1-2 \rho\right) & (\text { if } q=1) .
\end{array}\right.
$$

Komori, Matsumoto and Tsumura mentioned the existence of functional relations for $\zeta_{\mathfrak{s l}(5)}$ in [3, p. 163], but they did not give an explicit formula. Nakamura conjectured that some functional relations for the multi-variable version of Witten zeta-functions are obtained by using multiple Lerch value relations (see p. 552 in [7]). We note that Komori, Matsumoto and Tsumura obtained a functional relation for zeta-functions without any 0 index (see [3, Theorem 7.1]).

In this paper we study functional relations for $\zeta_{\mathfrak{s l}(3)}, \zeta_{\mathfrak{s l}(4)}$ and $\zeta_{\mathfrak{s l}(5)}$. In particular, we give an elementary proof of functional relations for these functions. Our results include functional relation (1.2) and a simpler form of (1.3) and (1.4). We guess that we can apply our method to $\zeta_{\mathfrak{s} l}(r+1)$ for all $r$ and zeta-functions without any 0 index.

\section{Statement of main results}

In this section we show the statement of our main results.

Let $k, l \in \mathbf{N}$ and $s, s_{1}, s_{2}, \ldots, s_{10}$ be complex variables. Let $R S\left(i_{1}, i_{2}, \ldots, i_{q}\right):=$ $\Re\left(s_{i_{1}}+s_{i_{2}}+\cdots+s_{i_{q}}\right)$ for $i_{1}, i_{2}, \ldots, i_{q} \in\{1,2, \ldots, 10\}$. Let

$$
\begin{aligned}
D_{2}= & \left\{s_{1} \in \mathbf{C} \mid R S(1)>1\right\}, \\
D_{2}^{\prime}= & \left\{\left(s_{1}, s_{2}\right) \in \mathbf{C}^{2} \mid R S(2)>1, R S(1,2)>2\right\}, \\
D_{3}= & \left\{\left(s_{1}, s_{2}, s_{3}\right) \in \mathbf{C}^{3} \mid R S(1,3)>1, R S(2,3)>1, R S(1,2,3)>2\right\}, \\
D_{4}= & \left\{\left(s_{1}, s_{2}, s_{3}, s_{4}, s_{5}, s_{6}\right) \in \mathbf{C}^{6} \mid\right. \\
& R S(3,5,6)>1, R S(1,4,6)>1, R S(2,4,5,6)>1, \\
& R S(2,3,4,5,6)>2, R S(1,3,4,5,6)>2, R S(1,2,4,5,6)>2, \\
& R S(1,2,3,4,5,6)>3\}, \\
D_{5}= & \left\{\left(s_{1}, s_{2}, s_{3}, s_{4}, s_{5}, s_{6}, s_{7}, s_{8}, s_{9}, s_{10}\right) \in \mathbf{C} 10 \mid\right. \\
& R S(4,7,9,10)>1, R S(3,6,7,8,9,10)>1, R S(2,5,6,8,9,10)>1, \\
& R S(1,5,8,10)>1, R S(3,4,6,7,8,9,10)>2, R S(1,2,5,6,8,9,10)>2, \\
& R S(2,4,5,6,7,8,9,10)>2, R S(2,3,5,6,7,8,9,10)>2, \\
& R S(1,4,5,7,8,9,10)>2, R S(1,3,5,6,7,8,9,10)>2, \\
& R S(2,3,4,5,6,7,8,9,10)>3, R S(1,3,4,5,6,7,8,9,10)>3,
\end{aligned}
$$


$R S(1,2,4,5,6,7,8,9,10)>3, R S(1,2,3,5,6,7,8,9,10)>3$,

$R S(1,2,3,4,5,6,7,8,9,10)>4\}$.

Let

$$
\begin{gathered}
K_{j}=K(k, l, j):=\left(\begin{array}{c}
k+l-j-1 \\
k-j
\end{array}\right) \\
L_{j}=L(k, l, j):=\left(\begin{array}{c}
k+l-j-1 \\
l-j
\end{array}\right) .
\end{gathered}
$$

The following theorems are our main results.

THEOREM 2.1. Let $f_{3}\left(s_{1}, s_{2}, s_{3}\right)$ be a complex function in the region $D_{3}$. Let $g(s)$ be a complex function in the region $D_{2}$ and $h\left(s_{1}, s_{2}\right)$ be a complex function in the region $D_{2}^{\prime}$. Assume that the following relations hold.

(a) $f_{3}\left(s_{1}, s_{2}, s_{3}\right)=f_{3}\left(s_{2}, s_{1}, s_{3}\right)$.

(b) $f_{3}\left(s_{1}, s_{2}, 0\right)=g\left(s_{1}\right) g\left(s_{2}\right)$.

(c) $f_{3}\left(s_{1}, 0, s_{2}\right)=h\left(s_{1}, s_{2}\right)$.

(d)

$$
\begin{aligned}
f_{3}(s, k, l)= & \sum_{j=2}^{k}(-1)^{k-j} K_{j} g(j) g(s+k+l-j) \\
& +\sum_{j=2}^{l}(-1)^{k} L_{j} h(s+k+l-j, j) \\
& +(-1)^{k-1} K_{1}(h(1, s+k+l-1)+g(s+k+l)) .
\end{aligned}
$$

(e)

$$
f_{3}(k, l, s)=\sum_{j=1}^{k} K_{j} h(j, s+k+l-j)+\sum_{j=1}^{l} L_{j} h(j, s+k+l-j) .
$$

(f) $h\left(s_{1}, s_{2}\right)+h\left(s_{2}, s_{1}\right)=g\left(s_{1}\right) g\left(s_{2}\right)-g\left(s_{1}+s_{2}\right)$.

Then we have

$$
\begin{aligned}
& (-1)^{k} f_{3}(s, k, l)+(-1)^{l} f_{3}(s, l, k)+f_{3}(k, l, s) \\
& =2 \sum_{j=1}^{[k / 2]}\left(\begin{array}{c}
k+l-2 j-1 \\
k-2 j
\end{array}\right) g(2 j) g(s+k+l-2 j) \\
& \quad+2 \sum_{j=1}^{[l / 2]}\left(\begin{array}{c}
k+l-2 j-1 \\
l-2 j
\end{array}\right) g(2 j) g(s+k+l-2 j) \\
& -\left(\left(\begin{array}{c}
k+l-1 \\
k
\end{array}\right)+\left(\begin{array}{c}
k+l-1 \\
l
\end{array}\right)\right) g(s+k+l) .
\end{aligned}
$$


In particular, we can take $f_{3}=\zeta_{\mathfrak{s l}(3)}, g=\zeta, h=\zeta_{2}$, where the function $\zeta_{2}$ is Euler double zeta-function

$$
\zeta_{2}\left(s_{1}, s_{2}\right)=\sum_{1 \leq m<n} \frac{1}{m^{s_{1}} n^{s_{2}}} .
$$

In this case relation (2.1) holds for all $s \in \mathbf{C}$ except for the singularities of each side of (2.1).

Note that we can obtain functional relation (1.2) by Theorem 2.1.

THEOREM 2.2. Let $f_{4}\left(s_{1}, s_{2}, s_{3}, s_{4}, s_{5}, s_{6}\right)$ be a complex function in the region $D_{4}$. Let $g(s)$ be a complex function in the region $D_{2}$ and $f_{3}\left(s_{1}, s_{2}, s_{3}\right)$ be a complex function in the region $\mathrm{D}_{3}$. Assume that the following relations hold.
(a) $f_{3}\left(s_{1}, s_{2}, s_{3}\right)=f_{3}\left(s_{2}, s_{1}, s_{3}\right)$.
(b) $f_{4}\left(s_{1}, s_{2}, s_{3}, s_{4}, s_{5}, s_{6}\right)=f_{4}\left(s_{3}, s_{2}, s_{1}, s_{5}, s_{4}, s_{6}\right)$.
(c) $f_{4}\left(s_{1}, s_{2}, s_{3}, s_{4}, 0,0\right)=g\left(s_{3}\right) f_{3}\left(s_{1}, s_{2}, s_{3}\right)$.
(d) $f_{4}\left(s_{1}, s_{2}, s_{4}, s_{3}, 0, s_{5}\right)=f_{4}\left(s_{2}, s_{1}, s_{4}, s_{3}, 0, s_{5}\right)$.
(e)

$$
\begin{aligned}
f_{4}\left(s_{1}, s_{2}, k, s_{3}, 0, l\right)= & \sum_{j=2}^{k}(-1)^{k-j} K_{j} g(j) f_{3}\left(s_{1}, s_{2}, s_{3}+k+l-j\right) \\
& +\sum_{j=2}^{l}(-1)^{k} L_{j} f_{4}\left(s_{1}, s_{2}, 0, s_{3}+k+l-j, 0, j\right) \\
& +(-1)^{k-1} K_{1} f_{4}\left(s_{1}, s_{2}, 1, s_{3}+k+l-2,0,1\right) .
\end{aligned}
$$

(f)

$$
\begin{aligned}
f_{4}\left(k, 0, s_{2}, s_{1}, l, s_{3}\right)= & \sum_{j=1}^{k} K_{j} f_{4}\left(j, 0, s_{2}, s_{1}, 0, s_{3}+k+l-j\right) \\
& +\sum_{j=1}^{l} L_{j} f_{4}\left(0,0, s_{2}, s_{1}, j, s_{3}+k+l-j\right) .
\end{aligned}
$$

(g)

$$
\begin{aligned}
& g(s) f_{3}\left(s_{1}, s_{2}, s_{3}\right) \\
& =f_{4}\left(s_{1}, s_{2}, 0, s_{3}, 0, s\right)+f_{4}\left(s_{1}, 0,0, s, s_{2}, s_{3}\right)+f_{4}\left(s, 0, s_{2}, s_{1}, 0, s_{3}\right) \\
& \quad+f_{3}\left(s_{1}, s_{2}, s_{3}+s\right)+f_{3}\left(s_{1}+s, s_{2}, s_{3}\right) .
\end{aligned}
$$

(h)

$$
\begin{aligned}
f_{4}\left(s_{1}, s_{2}, 1, s_{3}, 0,1\right)= & f_{4}\left(s_{1}, 0,0,1, s_{2}, s_{3}+1\right)+f_{4}\left(1,0, s_{2}, s_{1}, 0, s_{3}+1\right) \\
& +f_{3}\left(s_{1}, s_{2}, s_{3}+2\right)+f_{3}\left(s_{1}+1, s_{2}, s_{3}+1\right) .
\end{aligned}
$$


Then we have

$$
\begin{aligned}
& (-1)^{k} f_{4}\left(s_{1}, s_{2}, k, s_{3}, 0, l\right)+(-1)^{l} f_{4}\left(s_{1}, s_{2}, l, s_{3}, 0, k\right) \\
& +f_{4}\left(k, 0, s_{2}, s_{1}, l, s_{3}\right)+f_{4}\left(l, 0, s_{1}, s_{2}, k, s_{3}\right) \\
& =2 \sum_{j=1}^{[k / 2]}\left(\begin{array}{c}
k+l-2 j-1 \\
k-2 j
\end{array}\right) g(2 j) f_{3}\left(s_{1}, s_{2}, s_{3}+k+l-2 j\right) \\
& +2 \sum_{j=1}^{[l / 2]}\left(\begin{array}{c}
k+l-2 j-1 \\
l-2 j
\end{array}\right) g(2 j) f_{3}\left(s_{1}, s_{2}, s_{3}+k+l-2 j\right) \\
& -\sum_{j=1}^{k}\left(\begin{array}{c}
k+l-j-1 \\
k-j
\end{array}\right) f_{3}\left(s_{1}+j, s_{2}, s_{3}+k+l-j\right) \\
& -\sum_{j=1}^{l}\left(\begin{array}{c}
k+l-j-1 \\
l-j
\end{array}\right) f_{3}\left(s_{2}+j, s_{1}, s_{3}+k+l-j\right) \\
& -\left(\left(\begin{array}{c}
k+l-1 \\
k
\end{array}\right)+\left(\begin{array}{c}
k+l-1 \\
l
\end{array}\right)\right) f_{3}\left(s_{1}, s_{2}, s_{3}+k+l\right) .
\end{aligned}
$$

In particular, we can take $f_{4}=\zeta_{\mathfrak{s l}(4)}, f_{3}=\zeta_{\mathfrak{s l}(3)}, g=\zeta$. In this case relation (2.2) holds for all $\left(s_{1}, s_{2}, s_{3}\right) \in \mathbf{C}^{3}$ except for the singularities of each side of (2.2).

Note that we can obtain a simpler form of (1.3) and (1.4) by Theorem 2.2.

THEOREM 2.3. Let $f_{5}\left(s_{1}, s_{2}, s_{3}, s_{4}, s_{5}, s_{6}, s_{7}, s_{8}, s_{9}, s_{10}\right)$ be a complex function in the region $D_{5}$. Let $g(s)$ be a complex function in the region $D_{2}$ and $f_{4}\left(s_{1}, s_{2}, s_{3}, s_{4}, s_{5}, s_{6}\right)$ be a complex function in the region $D_{4}$. Assume that the following relations hold.

(a) $f_{4}\left(s_{1}, s_{2}, s_{3}, s_{4}, s_{5}, s_{6}\right)=f_{4}\left(s_{3}, s_{2}, s_{1}, s_{5}, s_{4}, s_{6}\right)$.

(b) $f_{5}\left(s_{1}, s_{2}, s_{3}, s_{4}, s_{5}, s_{6}, s_{7}, s_{8}, s_{9}, s_{10}\right)=f_{5}\left(s_{4}, s_{3}, s_{2}, s_{1}, s_{7}, s_{6}, s_{5}, s_{9}, s_{8}, s_{10}\right)$.

(c) $f_{5}\left(s_{1}, s_{2}, s_{3}, s_{4}, s_{5}, s_{6}, 0, s_{7}, 0,0\right)=g\left(s_{4}\right) f_{4}\left(s_{1}, s_{2}, s_{3}, s_{5}, s_{6}, s_{7}\right)$.

(d) $f_{5}\left(s_{1}, s_{2}, s_{3}, s_{9}, s_{4}, s_{5}, 0, s_{6}, 0, s_{10}\right)=f_{5}\left(s_{3}, s_{2}, s_{1}, s_{9}, s_{5}, s_{4}, 0, s_{6}, 0, s_{10}\right)$.

(e)

$$
\begin{aligned}
& f_{5}\left(s_{1}, s_{2}, s_{3}, k, s_{4}, s_{5}, 0, s_{6}, 0, l\right) \\
& =\sum_{j=2}^{k}(-1)^{k-j} K_{j} f_{5}\left(s_{1}, s_{2}, s_{3}, j, s_{4}, s_{5}, 0, s_{6}+k+l-j, 0,0\right) \\
& \quad+\sum_{j=2}^{l}(-1)^{k} L_{j} f_{5}\left(s_{1}, s_{2}, s_{3}, 0, s_{4}, s_{5}, 0, s_{6}+k+l-j, 0, j\right) \\
& \quad+(-1)^{k-1} K_{1} f_{5}\left(s_{1}, s_{2}, s_{3}, 1, s_{4}, s_{5}, 0, s_{6}+k+l-2,0,1\right) .
\end{aligned}
$$


(f)

$$
\begin{aligned}
& f_{5}\left(k, 0, s_{2}, s_{3}, s_{1}, 0, s_{5}, s_{4}, l, s_{6}\right) \\
& =\sum_{j=1}^{k} K_{j} f_{5}\left(j, 0, s_{2}, s_{3}, s_{1}, 0, s_{5}, s_{4}, 0, s_{6}+k+l-j\right) \\
& \quad+\sum_{j=1}^{l} L_{j} f_{5}\left(0,0, s_{2}, s_{3}, s_{1}, 0, s_{5}, s_{4}, j, s_{6}+k+l-j\right) .
\end{aligned}
$$

(g)

$$
\begin{aligned}
& f_{5}\left(s_{1}, 0,0, s_{3}, k, s_{2}, l, s_{4}, s_{5}, s_{6}\right) \\
& =\sum_{j=1}^{k} K_{j} f_{5}\left(s_{1}, 0,0, s_{3}, j, s_{2}, 0, s_{4}, s_{5}, s_{6}+k+l-j\right) \\
& \quad+\sum_{j=1}^{l} L_{j} f_{5}\left(s_{1}, 0,0, s_{3}, 0, s_{2}, j, s_{4}, s_{5}, s_{6}+k+l-j\right) .
\end{aligned}
$$

(h)

$$
\begin{aligned}
& g(s) f_{4}\left(s_{1}, s_{2}, s_{3}, s_{4}, s_{5}, s_{6}\right) \\
& =f_{5}\left(s_{1}, s_{2}, s_{3}, 0, s_{4}, s_{5}, 0, s_{6}, 0, s\right)+f_{5}\left(s_{1}, s_{2}, 0,0, s_{4}, 0, s_{3}, s, s_{5}, s_{6}\right) \\
& \quad+f_{5}\left(s_{1}, 0,0, s_{3}, s, s_{2}, 0, s_{4}, s_{5}, s_{6}\right)+f_{5}\left(s, 0, s_{2}, s_{3}, s_{1}, 0, s_{5}, s_{4}, 0, s_{6}\right) \\
& \quad+f_{4}\left(s_{1}, s_{2}, s_{3}, s_{4}, s_{5}, s_{6}+s\right)+f_{4}\left(s_{1}, s_{2}, s_{3}, s_{4}+s, s_{5}, s_{6}\right) \\
& \quad+f_{4}\left(s_{1}+s, s_{2}, s_{3}, s_{4}, s_{5}, s_{6}\right) .
\end{aligned}
$$

(i)

$$
\begin{aligned}
& f_{5}\left(s_{1}, s_{2}, s_{3}, 1, s_{4}, s_{5}, 0, s_{6}, 0,1\right) \\
& =f_{5}\left(s_{1}, s_{2}, 0,0, s_{4}, 0, s_{3}, 1, s_{5}, s_{6}+1\right)+f_{5}\left(s_{1}, 0,0, s_{3}, 1, s_{2}, 0, s_{4}, s_{5}, s_{6}+1\right) \\
& \quad+f_{5}\left(1,0, s_{2}, s_{3}, s_{1}, 0, s_{5}, s_{4}, 0, s_{6}+1\right)+f_{4}\left(s_{1}, s_{2}, s_{3}, s_{4}+1, s_{5}, s_{6}+1\right) \\
& \quad+f_{4}\left(s_{1}+1, s_{2}, s_{3}, s_{4}, s_{5}, s_{6}+1\right)+f_{4}\left(s_{1}, s_{2}, s_{3}, s_{4}, s_{5}, s_{6}+2\right) .
\end{aligned}
$$


Then we have

$$
\begin{aligned}
(-1)^{k} f_{5}\left(s_{1}, s_{2}, s_{3}, k, s_{4}, s_{5}, 0, s_{6}, 0, l\right)+(-1)^{l} f_{5}\left(s_{1}, s_{2}, s_{3}, l, s_{4}, s_{5}, 0, s_{6}, 0, k\right) \\
+f_{5}\left(k, 0, s_{2}, s_{3}, s_{1}, 0, s_{5}, s_{4}, l, s_{6}\right)+f_{5}\left(l, 0, s_{2}, s_{1}, s_{3}, 0, s_{4}, s_{5}, k, s_{6}\right) \\
+f_{5}\left(s_{1}, 0,0, s_{3}, k, s_{2}, l, s_{4}, s_{5}, s_{6}\right) \\
=2 \sum_{j=1}^{[k / 2]}\left(\begin{array}{c}
k+l-2 j-1 \\
k-2 j
\end{array}\right) g(2 j) f_{4}\left(s_{1}, s_{2}, s_{3}, s_{4}, s_{5}, s_{6}+k+l-2 j\right) \\
+2 \sum_{j=1}^{[l / 2]}\left(\begin{array}{c}
k+l-2 j-1 \\
l-2 j
\end{array}\right) g(2 j) f_{4}\left(s_{1}, s_{2}, s_{3}, s_{4}, s_{5}, s_{6}+k+l-2 j\right) \\
-\sum_{j=1}^{k}\left(\begin{array}{c}
k+l-j-1 \\
k-j
\end{array}\right)\left(f_{4}\left(s_{1}, s_{2}, s_{3}, s_{4}+j, s_{5}, s_{6}+k+l-j\right)\right. \\
\left.\quad+f_{4}\left(s_{1}+j, s_{2}, s_{3}, s_{4}, s_{5}, s_{6}+k+l-j\right)\right) \\
-\sum_{j=1}^{l}\left(\begin{array}{c}
k+l-j-1 \\
l-j
\end{array}\right)\left(f_{4}\left(s_{3}, s_{2}, s_{1}, s_{5}+j, s_{4}, s_{6}+k+l-j\right)\right. \\
\left.\quad+f_{4}\left(s_{3}+j, s_{2}, s_{1}, s_{5}, s_{4}, s_{6}+k+l-j\right)\right) \\
\left.-\left(\begin{array}{c}
k+l-1 \\
l
\end{array}\right)+\left(\begin{array}{c}
k+l-1 \\
k
\end{array}\right)\right) f_{4}\left(s_{1}, s_{2}, s_{3}, s_{4}, s_{5}, s_{6}+k+l\right)
\end{aligned}
$$

In particular, we can take $f_{5}=\zeta_{\mathfrak{s l}(5)}, f_{4}=\zeta_{\mathfrak{s l}(4)}, g=\zeta$. In this case relation (2.3) holds for all $\left(s_{1}, s_{2}, s_{3}, s_{4}, s_{5}, s_{6}\right) \in \mathbf{C}^{6}$ except for the singularities of each side of (2.3).

In [1] Bradley, Cai and Zhou stated that

$$
\begin{aligned}
& (-1)^{k} \zeta_{\mathfrak{s l}(4)}\left(s_{1}, s_{2}, k, s_{3}, 0, l\right)+(-1)^{l} \zeta_{\mathfrak{s l}(4)}\left(s_{1}, s_{2}, l, s_{3}, 0, k\right) \\
& +\zeta_{\mathfrak{s l}(4)}\left(k, 0, s_{2}, s_{1}, l, s_{3}\right)+\zeta_{\mathfrak{s l}(4)}\left(l, 0, s_{1}, s_{2}, k, s_{3}\right) \\
& =\sum_{i=1}^{\max (k, l)}\left(\left(\begin{array}{c}
k+l-i-1 \\
k-1
\end{array}\right)+\left(\begin{array}{c}
k+l-i-1 \\
l-1
\end{array}\right)\right)(-1)^{i} \zeta(i) \\
& \times \zeta_{\mathfrak{s l}(3)}\left(s_{1}, s_{2}, s_{3}+k+l-i\right) \\
& +\sum_{i=1}^{k}\left(\begin{array}{c}
k+l-i-1 \\
l-1
\end{array}\right)\left\{\zeta(i) \zeta_{\mathfrak{s l}(3)}\left(s_{1}, s_{2}, s_{3}+k+l-i\right)\right. \\
& \left.-\zeta_{\mathfrak{s l}(3)}\left(s_{1}+i, s_{2}, s_{3}+k+l-i\right)-\zeta_{\mathfrak{s l}(3)}\left(s_{1}, s_{2}, s_{3}+k+l\right)\right\}
\end{aligned}
$$

holds, but the right hand side of (2.4) includes the factor $\zeta(1)$. Hence, strictly speaking, relation (2.4) is not valid. By Theorem 2.2 we obtain a functional relation which does not 
include $\zeta(1)$. The proof of our theorems are inspired by [1] and based on some elementary calculations.

Since we can take

$$
f_{3}\left(s_{1}, s_{2}, s_{3}\right)=\frac{\sin \left(2 \pi s_{1}\right) \sin \left(2 \pi s_{2}\right) \sin \left(2 \pi s_{3}\right)}{a^{s_{1}+s_{2}}(2 a)^{s_{3}}}, \quad g=h=0
$$

in Theorem 2.1,

$$
f_{4}\left(s_{1}, s_{2}, s_{3}, s_{4}, s_{4}, s_{6}\right)=\frac{\sin \left(2 \pi s_{1}\right) \sin \left(2 \pi s_{2}\right) \ldots \sin \left(2 \pi s_{6}\right)}{a^{s_{1}+s_{2}+s_{3}}(2 a)^{s_{4}+s_{5}}(3 a)^{s_{6}}}, \quad f_{3}=g=0
$$

in Theorem 2.2 and

$$
\begin{aligned}
& f_{5}\left(s_{1}, s_{2}, s_{3}, s_{4}, s_{5}, s_{6}, s_{7}, s_{8}, s_{9}, s_{10}\right)=\frac{\sin \left(2 \pi s_{1}\right) \ldots \sin \left(2 \pi s_{10}\right)}{a^{s_{1}+s_{2}+s_{3}+s_{4}}(2 a)^{s_{5}+s_{6}+s_{7}}(3 a)^{s_{8}+s_{9}}(4 a)^{s_{10}}}, \\
& f_{4}=g=0
\end{aligned}
$$

in Theorem 2.3, where $a>0$, we can not characterize $\zeta_{\mathfrak{s l}(3)}, \zeta_{\mathfrak{s l}(4)}$ and $\zeta_{\mathfrak{s l}(5)}$ by the functional relations (2.1), (2.2) and (2.3), respectively.

\section{Lemmas for the proof of Theorems}

In this section, we collect some auxiliary results and definitions.

Let $N_{0}=0$. By (1.1) we have

$$
\zeta_{\mathfrak{s} l(r+1)}(\mathbf{s})=\sum_{1 \leq N_{1}<\cdots<N_{r}} \prod_{j=1}^{r} \prod_{k=1}^{r-j+1}\left(N_{j+k-1}-N_{k-1}\right)^{-s_{j, k}} .
$$

Note that we have

$$
\begin{aligned}
& \zeta_{\mathfrak{s l}(2)}\left(s_{1}\right)=\zeta\left(s_{1}\right), \\
& \zeta_{\mathfrak{s l}(3)}\left(s_{1}, s_{2}, s_{3}\right)=\sum_{n_{1}, n_{2}=1}^{\infty} \frac{1}{n_{1}^{s_{1}} n_{2}^{s_{2}}\left(n_{1}+n_{2}\right)^{s_{3}}}=\sum_{1 \leq N_{1}<N_{2}} \frac{1}{N_{1}^{s_{1}}\left(N_{2}-N_{1}\right)^{s_{2}} N_{2}^{s_{3}}}, \\
& \zeta_{\mathfrak{s l}(4)}\left(s_{1}, s_{2}, s_{3}, s_{4}, s_{5}, s_{6}\right) \\
& =\sum_{n_{1}, n_{2}, n_{3}=1}^{\infty} \frac{1}{n_{1}^{s_{1}} n_{2}^{s_{2}} n_{3}^{s_{3}}\left(n_{1}+n_{2}\right)^{s_{4}}\left(n_{2}+n_{3}\right)^{s_{5}}\left(n_{1}+n_{2}+n_{3}\right)^{s_{6}}} \\
& =\sum_{1 \leq N_{1}<N_{2}<N_{3}} \frac{1}{N_{1}^{S_{1}}\left(N_{2}-N_{1}\right)^{s_{2}}\left(N_{3}-N_{2}\right)^{s_{3}} N_{2}^{s_{4}}\left(N_{3}-N_{1}\right)^{s_{5}} N_{3}^{s_{6}}}
\end{aligned}
$$


and

$$
\begin{aligned}
& \zeta_{\mathfrak{s l}(5)}\left(s_{1}, s_{2}, s_{3}, s_{4}, s_{5}, s_{6}, s_{7}, s_{8}, s_{9}, s_{10}\right) \\
& =\sum_{n_{1}, n_{2}, n_{3}, n_{4}=1}^{\infty} \frac{1}{n_{1}^{s_{1}} n_{2}^{s_{2}} n_{3}^{s_{3}} n_{4}^{s_{4}}\left(n_{1}+n_{2}\right)^{s_{5}}\left(n_{2}+n_{3}\right)^{s_{6}}\left(n_{3}+n_{4}\right)^{s_{7}}} \\
& \quad \times \frac{1}{\left(n_{1}+n_{2}+n_{3}\right)^{s_{8}}\left(n_{2}+n_{3}+n_{4}\right)^{s_{9}}\left(n_{1}+n_{2}+n_{3}+n_{4}\right)^{s_{10}}} \\
& =\sum_{1 \leq N_{1}<N_{2}<N_{3}<N_{4}} \frac{1}{N_{1}^{s_{1}}\left(N_{2}-N_{1}\right)^{s_{2}}\left(N_{3}-N_{2}\right)^{s_{3}}\left(N_{4}-N_{3}\right)^{s_{4}} N_{2}^{s_{5}}} \\
& \quad \times \frac{1}{\left(N_{3}-N_{1}\right)^{s_{6}}\left(N_{4}-N_{2}\right)^{s_{7}} N_{3}^{s_{8}}\left(N_{4}-N_{1}\right)^{s_{9}} N_{4}^{s_{10}}} .
\end{aligned}
$$

By elementary calculations, we can check that the sum (3.1) (resp. (3.2), (3.3)) is absolutely convergent in the region $D_{3}$ (resp. $D_{4}, D_{5}$ ).

The following formulas are similar to the harmonic product formulas for Euler-Zagier multiple zeta-functions. The method of the proof of lemmas can be used for general cases. For example, $\zeta(s) \zeta_{\mathfrak{s l}(5)}\left(s_{1}, s_{2}, s_{3}, s_{4}, s_{5}, s_{6}, s_{7}, s_{8}, s_{9}, s_{10}\right), \zeta_{\mathfrak{s l}(3)}\left(s_{1}, s_{2}, s_{3}\right) \zeta_{\mathfrak{s l}(3)}\left(s_{4}, s_{5}, s_{6}\right)$, etc.

LEMMA 3.1 (see p. 5 [1]). We have

$$
\begin{aligned}
& \zeta(s) \zeta_{\mathfrak{s l}(3)}\left(s_{1}, s_{2}, s_{3}\right) \\
& =\zeta_{\mathfrak{s l}(4)}\left(s_{1}, s_{2}, 0, s_{3}, 0, s\right)+\zeta_{\mathfrak{s l}(4)}\left(s_{1}, 0,0, s, s_{2}, s_{3}\right)+\zeta_{\mathfrak{s l}(4)}\left(s, 0, s_{2}, s_{1}, 0, s_{3}\right) \\
& \quad+\zeta_{\mathfrak{s l}(3)}\left(s_{1}, s_{2}, s_{3}+s\right)+\zeta_{\mathfrak{s l}(3)}\left(s_{1}+s, s_{2}, s_{3}\right) .
\end{aligned}
$$

PROOF. This lemma was used in p. 5 [1], but we give a new proof. We have

$$
\begin{aligned}
& \zeta(s) \zeta_{\mathfrak{s} l(3)}\left(s_{1}, s_{2}, s_{3}\right)=\sum_{N_{3}=1}^{\infty} \frac{1}{N_{3}^{s}} \sum_{1 \leq N_{1}<N_{2}} \frac{1}{N_{1}^{s_{1}}\left(N_{2}-N_{1}\right)^{s_{2} N_{2}^{s_{3}}}} \\
& =\left(\sum_{1 \leq N_{1}<N_{2}<N_{3}}+\sum_{1 \leq N_{1}<N_{3}<N_{2}}+\sum_{1 \leq N_{3}<N_{1}<N_{2}}+\sum_{1 \leq N_{1}<N_{2}=N_{3}}\right. \\
& \left.\quad+\sum_{1 \leq N_{1}=N_{3}<N_{2}}\right) \frac{1}{N_{1}^{s_{1}}\left(N_{2}-N_{1}\right)^{s_{2}} N_{2}^{s_{3}} N_{3}^{s}} \\
& =\sum_{n_{1}, n_{2}, n_{3}=1}^{\infty} \frac{1}{n_{1}^{s_{1}} n_{2}^{S_{2}}\left(n_{1}+n_{2}\right)^{s_{3}}\left(n_{1}+n_{2}+n_{3}\right)^{s}} \\
& +\sum_{n_{1}, n_{2}, n_{3}=1}^{\infty} \frac{1}{n_{1}^{s_{1}}\left(n_{2}+n_{3}\right)^{s_{2}}\left(n_{1}+n_{2}+n_{3}\right)^{s_{3}}\left(n_{1}+n_{2}\right)^{s}}
\end{aligned}
$$




$$
\begin{aligned}
& \quad+\sum_{n_{1}, n_{2}, n_{3}=1}^{\infty} \frac{1}{\left(n_{1}+n_{2}\right)^{s_{1}} n_{3}^{s_{2}}\left(n_{1}+n_{2}+n_{3}\right)^{s_{3}} n_{1}^{s}} \\
& +\zeta_{\mathfrak{s l}(3)}\left(s_{1}, s_{2}, s_{3}+s\right)+\zeta_{\mathfrak{s l}(3)}\left(s_{1}+s, s_{2}, s_{3}\right) \\
& =\zeta_{\mathfrak{s l}(4)}\left(s_{1}, s_{2}, 0, s_{3}, 0, s\right)+\zeta_{\mathfrak{s l}(4)}\left(s_{1}, 0,0, s, s_{2}, s_{3}\right)+\zeta_{\mathfrak{s l}(4)}\left(s, 0, s_{2}, s_{1}, 0, s_{3}\right) \\
& +\zeta_{\mathfrak{s l}(3)}\left(s_{1}, s_{2}, s_{3}+s\right)+\zeta_{\mathfrak{s l}(3)}\left(s_{1}+s, s_{2}, s_{3}\right) .
\end{aligned}
$$

LEMMA 3.2. We have

$$
\begin{aligned}
& \zeta(s) \zeta_{\mathfrak{s l}(4)}\left(s_{1}, s_{2}, s_{3}, s_{4}, s_{5}, s_{6}\right) \\
& =\zeta_{\mathfrak{s l}(5)}\left(s_{1}, s_{2}, s_{3}, 0, s_{4}, s_{5}, 0, s_{6}, 0, s\right)+\zeta_{\mathfrak{s l}(5)}\left(s_{1}, s_{2}, 0,0, s_{4}, 0, s_{3}, s, s_{5}, s_{6}\right) \\
& +\zeta_{\mathfrak{s l}(5)}\left(s_{1}, 0,0, s_{3}, s, s_{2}, 0, s_{4}, s_{5}, s_{6}\right)+\zeta_{\mathfrak{s l}(5)}\left(s, 0, s_{2}, s_{3}, s_{1}, 0, s_{5}, s_{4}, 0, s_{6}\right) \\
& +\zeta_{\mathfrak{s l}(4)}\left(s_{1}, s_{2}, s_{3}, s_{4}, s_{5}, s_{6}+s\right)+\zeta_{\mathfrak{s l}(4)}\left(s_{1}, s_{2}, s_{3}, s_{4}+s, s_{5}, s_{6}\right) \\
& +\zeta_{\mathfrak{s l}(4)}\left(s_{1}+s, s_{2}, s_{3}, s_{4}, s_{5}, s_{6}\right) \text {. }
\end{aligned}
$$

Proof. We have

$$
\begin{aligned}
& \zeta(s) \zeta_{\mathfrak{s l}(4)}\left(s_{1}, s_{2}, s_{3}, s_{4}, s_{5}, s_{6}\right) \\
& =\sum_{N_{4}=1}^{\infty} \frac{1}{N_{4}^{s}} \sum_{1 \leq N_{1}<N_{2}<N_{3}} \frac{1}{N_{1}^{s_{1}}\left(N_{2}-N_{1}\right)^{s_{2}}\left(N_{3}-N_{2}\right)^{s_{3}} N_{2}^{s_{4}}\left(N_{3}-N_{1}\right)^{s_{5}} N_{3}^{s_{6}}} \\
& =\left(\sum_{1 \leq N_{1}<N_{2}<N_{3}<N_{4}}+\sum_{1 \leq N_{1}<N_{2}<N_{4}<N_{3}}+\sum_{1 \leq N_{1}<N_{4}<N_{2}<N_{3}}\right. \\
& +\sum_{1 \leq N_{4}<N_{1}<N_{2}<N_{3}}+\sum_{1 \leq N_{1}<N_{2}<N_{3}=N_{4}}+\sum_{1 \leq N_{1}<N_{2}=N_{4}<N_{3}} \\
& \left.+\sum_{1 \leq N_{1}=N_{4}<N_{2}<N_{3}}\right) \frac{1}{N_{1}^{s_{1}}\left(N_{2}-N_{1}\right)^{s_{2}}\left(N_{3}-N_{2}\right)^{s_{3}} N_{2}^{s_{4}}\left(N_{3}-N_{1}\right)^{s_{5}} N_{3}^{s_{6}} N_{4}^{s}} \\
& =\sum_{n_{1}, n_{2}, n_{3} n_{4}=1}^{\infty} \frac{1}{n_{1}^{s_{1}} n_{2}^{s_{2}} n_{3}^{s_{3}}\left(n_{1}+n_{2}\right)^{s_{4}}\left(n_{2}+n_{3}\right)^{s_{5}}\left(n_{1}+n_{2}+n_{3}\right)^{s_{6}}\left(n_{1}+n_{2}+n_{3}+n_{4}\right)^{s}} \\
& +\sum_{n_{1}, n_{2}, n_{3} n_{4}=1}^{\infty} \frac{1}{n_{1}^{s_{1}} n_{2}^{s_{2}}\left(n_{3}+n_{4}\right)^{s_{3}}\left(n_{1}+n_{2}\right)^{s_{4}}} \\
& \times \frac{1}{\left(n_{2}+n_{3}+n_{4}\right)^{s_{5}}\left(n_{1}+n_{2}+n_{3}+n_{4}\right)^{s_{6}}\left(n_{1}+n_{2}+n_{3}\right)^{s}}
\end{aligned}
$$




$$
\begin{aligned}
& +\sum_{n_{1}, n_{2}, n_{3} n_{4}=1}^{\infty} \frac{1}{n_{1}^{s_{1}}\left(n_{2}+n_{3}\right)^{s_{2}} n_{4}^{s_{3}}\left(n_{1}+n_{2}+n_{3}\right)^{s_{4}}} \\
& \times \frac{1}{\left(n_{2}+n_{3}+n_{4}\right)^{s_{5}}\left(n_{1}+n_{2}+n_{3}+n_{4}\right)^{s_{6}}\left(n_{1}+n_{2}\right)^{s}} \\
& +\sum_{n_{1}, n_{2}, n_{3} n_{4}=1}^{\infty} \frac{1}{\left(n_{1}+n_{2}\right)^{s_{1}} n_{3}^{s_{2}} n_{4}^{s_{3}}\left(n_{1}+n_{2}+n_{3}\right)^{s_{4}}\left(n_{3}+n_{4}\right)^{s_{5}}\left(n_{1}+n_{2}+n_{3}+n_{4}\right)^{s_{6}} n_{1}^{s}} \\
& +\zeta_{\mathfrak{s l}(4)}\left(s_{1}, s_{2}, s_{3}, s_{4}, s_{5}, s_{6}+s\right)+\zeta_{\mathfrak{s l}(4)}\left(s_{1}, s_{2}, s_{3}, s_{4}+s, s_{5}, s_{6}\right) \\
& +\zeta_{\mathfrak{s l}(4)}\left(s_{1}+s, s_{2}, s_{3}, s_{4}, s_{5}, s_{6}\right) \\
& =\zeta_{\mathfrak{s l}(5)}\left(s_{1}, s_{2}, s_{3}, 0, s_{4}, s_{5}, 0, s_{6}, 0, s\right)+\zeta_{\mathfrak{s l}(5)}\left(s_{1}, s_{2}, 0,0, s_{4}, 0, s_{3}, s, s_{5}, s_{6}\right) \\
& +\zeta_{\mathfrak{s l}(5)}\left(s_{1}, 0,0, s_{3}, s, s_{2}, 0, s_{4}, s_{5}, s_{6}\right)+\zeta_{\mathfrak{s l}(5)}\left(s, 0, s_{2}, s_{3}, s_{1}, 0, s_{5}, s_{4}, 0, s_{6}\right) \\
& +\zeta_{\mathfrak{s l}(4)}\left(s_{1}, s_{2}, s_{3}, s_{4}, s_{5}, s_{6}+s\right)+\zeta_{\mathfrak{s l}(4)}\left(s_{1}, s_{2}, s_{3}, s_{4}+s, s_{5}, s_{6}\right) \\
& +\zeta_{\mathfrak{s l}(4)}\left(s_{1}+s, s_{2}, s_{3}, s_{4}, s_{5}, s_{6}\right) \text {. }
\end{aligned}
$$

The following lemma is a kind of partial fraction decompositions.

Lemma 3.3. Let $k, l \in \mathbf{N}, a \in \mathbf{R} \backslash\{0\}$. Then we have

$$
\frac{1}{x^{k}(x+a)^{l}}=\sum_{j=1}^{k} \frac{c_{j}}{x^{j}}+\sum_{i=1}^{l} \frac{d_{i}}{(x+a)^{i}},
$$

where

$$
c_{j}=(-1)^{k-j}\left(\begin{array}{c}
l+k-j-1 \\
k-j
\end{array}\right) a^{-l-k+j}
$$

and

$$
d_{j}=(-1)^{k}\left(\begin{array}{c}
l+k-j-1 \\
l-j
\end{array}\right) a^{-l-k+j} .
$$

Proof. Let $f(x)=x^{-k}(x+a)^{-l}$. By Lemma 1 we have

$$
\begin{aligned}
c_{j} & =\frac{1}{(k-j) !} \lim _{x \rightarrow 0} \frac{d^{k-j}}{d x^{k-j}} x^{k} f(x) \\
& =\frac{1}{(k-j) !} \lim _{x \rightarrow 0}(-1)^{k-j} \frac{(l+k-j-1) !}{(l-1) !}(x+a)^{-l-k+j} \\
& =(-1)^{k-j} \frac{(l+k-j-1) !}{(k-j) !(l-1) !} a^{-l-k+j}
\end{aligned}
$$




$$
=(-1)^{k-j}\left(\begin{array}{c}
l+k-j-1 \\
k-j
\end{array}\right) a^{-l-k+j}
$$

and

$$
\begin{aligned}
d_{j} & =\frac{1}{(l-j) !} \lim _{x \rightarrow-a} \frac{d^{l-j}}{d x^{l-j}}(x+a)^{l} f(x) \\
& =\frac{1}{(l-j) !} \lim _{x \rightarrow-a}(-1)^{l-j} \frac{(l+k-j-1) !}{(k-1) !} x^{-l-k+j} \\
& =(-1)^{k} \frac{(l+k-j-1) !}{(l-j) !(k-1) !} a^{-l-k+j} \\
& =(-1)^{k}\left(\begin{array}{c}
l+k-j-1 \\
l-j
\end{array}\right) a^{-l-k+j} .
\end{aligned}
$$

The following lemmas are important in the proof of Theorem 2.3.

\section{LEMMA 3.4. Let $k, l \in \mathbf{N}$. We have}

$$
\begin{aligned}
\zeta_{\mathfrak{s l}(5)}\left(s_{1}, s_{2}, s_{3}, k, s_{4}, s_{5}, 0, s_{6}, 0, l\right)= & \sum_{j=2}^{k}(-1)^{k-j} K_{j} \zeta_{\mathfrak{s l}(5)}\left(s_{1}, s_{2}, s_{3}, j, s_{4}, s_{5}, 0, s_{6}+k+l-j, 0,0\right) \\
& +\sum_{j=2}^{l}(-1)^{k} L_{j} \zeta_{\mathfrak{s l}(5)}\left(s_{1}, s_{2}, s_{3}, 0, s_{4}, s_{5}, 0, s_{6}+k+l-j, 0, j\right) \\
& +E_{5}\left(s_{1}, s_{2}, s_{3}, s_{4}, s_{5}, s_{6}, k, l\right)
\end{aligned}
$$

where

$E_{5}\left(s_{1}, s_{2}, s_{3}, s_{4}, s_{5}, s_{6}, k, l\right)=(-1)^{k-1} K_{1} \zeta_{\mathfrak{s} l(5)}\left(s_{1}, s_{2}, s_{3}, 1, s_{4}, s_{5}, 0, s_{6}+k+l-2,0,1\right)$

$=(-1)^{k-1} K_{1}\left(\zeta_{\mathfrak{s} l(5)}\left(s_{1}, s_{2}, 0,0, s_{4}, 0, s_{3}, 1, s_{5}, s_{6}+k+l-1\right)\right.$

$+\zeta_{\mathfrak{s l}(5)}\left(s_{1}, 0,0, s_{3}, 1, s_{2}, 0, s_{4}, s_{5}, s_{6}+k+l-1\right)+\zeta_{\mathfrak{s l}(5)}\left(1,0, s_{2}, s_{3}, s_{1}, 0, s_{5}, s_{4}, 0, s_{6}+k+l-1\right)$

$+\zeta_{\mathfrak{s l}(4)}\left(s_{1}, s_{2}, s_{3}, s_{4}+1, s_{5}, s_{6}+k+l-1\right)+\zeta_{\mathfrak{s l}(4)}\left(s_{1}+1, s_{2}, s_{3}, s_{4}, s_{5}, s_{6}+k+l-1\right)$

$\left.+\zeta_{\mathfrak{s l}(4)}\left(s_{1}, s_{2}, s_{3}, s_{4}, s_{5}, s_{6}+k+l\right)\right)$.

We have

$$
\begin{aligned}
\zeta_{\mathfrak{s l}(5)}\left(k, 0, s_{2}, s_{3}, s_{1}, 0, s_{5}, s_{4}, l, s_{6}\right) & =\sum_{j=1}^{k} K_{j} \zeta_{\mathfrak{s l}(5)}\left(j, 0, s_{2}, s_{3}, s_{1}, 0, s_{5}, s_{4}, 0, s_{6}+k+l-j\right) \\
& +\sum_{j=1}^{l} L_{j} \zeta_{\mathfrak{s l}(5)}\left(0,0, s_{2}, s_{3}, s_{1}, 0, s_{5}, s_{4}, j, s_{6}+k+l-j\right)
\end{aligned}
$$


and

$\begin{aligned} \zeta_{\mathfrak{s l}(5)}\left(s_{1}, 0,0, s_{3}, k, s_{2}, l, s_{4}, s_{5}, s_{6}\right) & =\sum_{j=1}^{k} K_{j} \zeta_{\mathfrak{s l}(5)}\left(s_{1}, 0,0, s_{3}, j, s_{2}, 0, s_{4}, s_{5}, s_{6}+k+l-j\right) \\ & +\sum_{j=1}^{l} L_{j} \zeta_{\mathfrak{s l}(5)}\left(s_{1}, 0,0, s_{3}, 0, s_{2}, j, s_{4}, s_{5}, s_{6}+k+l-j\right) .\end{aligned}$

PROOF. Note that $\left(\begin{array}{c}k+l-2 \\ k-1\end{array}\right)=\left(\begin{array}{c}k+l-2 \\ l-1\end{array}\right)$. By Lemma 3.3 we have $\zeta_{\mathfrak{s l}(5)}\left(s_{1}, s_{2}, s_{3}, k, s_{4}, s_{5}, 0, s_{6}, 0, l\right)$

$$
\begin{aligned}
& =\sum_{n_{1}, n_{2}, n_{3}, n_{4}=1}^{\infty} \frac{1}{n_{1}^{s_{1}} n_{2}^{s_{2}} n_{3}^{s_{3}}\left(n_{1}+n_{2}\right)^{s_{4}}\left(n_{2}+n_{3}\right)^{s_{5}}\left(n_{1}+n_{2}+n_{3}\right)^{s_{6}} n_{4}^{k}\left(n_{1}+n_{2}+n_{3}+n_{4}\right)^{l}} \\
& =\sum_{j=2}^{k}(-1)^{k-j} K_{j} \zeta_{\mathfrak{s l}(5)}\left(s_{1}, s_{2}, s_{3}, j, s_{4}, s_{5}, 0, s_{6}+k+l-j, 0,0\right) \\
& +\sum_{j=2}^{l}(-1)^{k} L_{j} \zeta_{\mathfrak{s l}(5)}\left(s_{1}, s_{2}, s_{3}, 0, s_{4}, s_{5}, 0, s_{6}+k+l-j, 0, j\right) \\
& +(-1)^{k-1} K_{1} \zeta_{\mathfrak{s l}(5)}\left(s_{1}, s_{2}, s_{3}, 1, s_{4}, s_{5}, 0, s_{6}+k+l-2,0,1\right) .
\end{aligned}
$$

By the method similar to the proof of Lemma 3.2, we have

$$
\begin{aligned}
& \zeta_{\mathfrak{s l}(5)}\left(s_{1}, s_{2}, s_{3}, 1, s_{4}, s_{5}, 0, s_{6}+k+l-2,0,1\right) \\
& =\sum_{n_{1}, n_{2}, n_{3}=1}^{\infty} \frac{1}{n_{1}^{s_{1}} n_{2}^{s_{2}} n_{3}^{s_{3}}\left(n_{1}+n_{2}\right)^{s_{4}}\left(n_{2}+n_{3}\right)^{s_{5}}\left(n_{1}+n_{2}+n_{3}\right)^{s_{6}+k+l-2}} \\
& \quad \times \frac{1}{n_{1}+n_{2}+n_{3}} \sum_{n_{4}=1}^{\infty}\left(\frac{1}{n_{4}}-\frac{1}{n_{1}+n_{2}+n_{3}+n_{4}}\right) \\
& =\sum_{n_{1}, n_{2}, n_{3}=1}^{\infty} \frac{1}{n_{1}^{s_{1}} n_{2}^{s_{2}} n_{3}^{s_{3}}\left(n_{1}+n_{2}\right)^{s_{4}\left(n_{2}+n_{3}\right)^{s_{5}}\left(n_{1}+n_{2}+n_{3}\right)^{s_{6}+k+l-1}}} \\
& \quad \times\left(\sum_{1 \leq n_{4}<n_{1}+n_{2}+n_{3}} \frac{1}{n_{4}}+\frac{1}{n_{1}+n_{2}+n_{3}}\right) \\
& =\sum_{1 \leq N_{1}<N_{2}<N_{3}} \frac{1}{N_{1}^{s_{1}}\left(N_{2}-N_{1}\right)^{s_{2}}\left(N_{3}-N_{2}\right)^{s_{3}} N_{2}^{s_{4}}\left(N_{3}-N_{1}\right)^{s_{5}} N_{3}^{s_{6}+k+l-1}} \times\left(\sum_{1 \leq N_{4}<N_{3}} \frac{1}{N_{4}}\right) \\
& +\zeta_{\mathfrak{s l}(4)}\left(s_{1}+1, s_{2}, s_{3}, s_{4}, s_{5}, s_{6}+k+l\right)
\end{aligned}
$$




$$
\begin{aligned}
& \left.=\left(\sum_{1 \leq N_{1}<N_{2}<N_{4}<N_{3}}+\sum_{1 \leq N_{1}<N_{4}<N_{2}<N_{3}}+\sum_{1 \leq N_{4}<N_{1}<N_{2}<N_{3}}+\sum_{1 \leq N_{1}<N_{2}=N_{4}<N_{3}}\right) \frac{1}{N_{1 \leq N_{1}=N_{4}<N_{2}<N_{3}}}\right) \\
& +\zeta_{\mathfrak{s l}(4)}\left(s_{1}, s_{2}, s_{3}, s_{4}, s_{5}, s_{6}+k+l\right) \\
& =\zeta_{\mathfrak{s l}(5)}\left(s_{1}, s_{2}, 0,0, s_{4}, 0, s_{3}, 1, s_{5}, s_{6}+k+l-1\right)+\zeta_{\mathfrak{s l}(5)}\left(s_{1}, 0,0, s_{3}, 1, s_{2}, 0, s_{4}, s_{5}, s_{6}+k+l-1\right) \\
& +\zeta_{\mathfrak{s l}(5)}\left(1,0, s_{2}, s_{3}, s_{1}, 0, s_{5}, s_{4}, 0, s_{6}+k+l-1\right)+\zeta_{\mathfrak{s l}(4)}\left(s_{1}, s_{2}, s_{3}, s_{4}+1, s_{5}^{s_{4}}\left(N_{3}-s_{6}+k+l-1\right)\right. \\
& +\zeta_{\mathfrak{s l}(4)}\left(s_{1}+1, s_{2}, s_{3}, s_{4}, s_{5}, s_{6}+k+l-1\right)+\zeta_{\mathfrak{s l}(4)}\left(s_{1}, s_{2}, s_{3}, s_{4}, s_{5}, s_{6}+k+l\right) .
\end{aligned}
$$

Similarly we have

$$
\begin{aligned}
& \zeta_{\mathfrak{s l}(5)}\left(k, 0, s_{2}, s_{3}, s_{1}, 0, s_{5}, s_{4}, l, s_{6}\right) \\
& =\sum_{n_{1}, n_{2}, n_{3}, n_{4}=1}^{\infty} \frac{1}{n_{3}^{s_{2}} n_{4}^{s_{3}}\left(n_{1}+n_{2}\right)^{s_{1}}\left(n_{3}+n_{4}\right)^{s_{5}}\left(n_{1}+n_{2}+n_{3}\right)^{s_{4}}\left(n_{1}+n_{2}+n_{3}+n_{4}\right)^{s_{6}}} \\
& =\sum_{j=1}^{k} K_{j} \zeta_{\mathfrak{s l}(5)}\left(j, 0, s_{2}, s_{3}, s_{1}, 0, s_{5}, s_{4}, 0, s_{6}+k+l-j\right) \\
& +\sum_{j=1}^{l} L_{j} \zeta_{\mathfrak{s l}(5)}\left(0,0, s_{2}, s_{3}, s_{1}, 0, s_{5}, s_{4}, j, s_{6}+k+l-j\right)
\end{aligned}
$$

and

$$
\begin{aligned}
& \zeta_{\mathfrak{s l}(5)}\left(s_{1}, 0,0, s_{3}, k, s_{2}, l, s_{4}, s_{5}, s_{6}\right) \\
& =\sum_{n_{1}, n_{2}, n_{3}, n_{4}=1}^{\infty} \frac{1}{n_{1}^{s_{1}} n_{4}^{s_{3}}\left(n_{2}+n_{3}\right)^{s_{2}}\left(n_{1}+n_{2}+n_{3}\right)^{s_{4}}\left(n_{2}+n_{3}+n_{4}\right)^{s_{5}}\left(n_{1}+n_{2}+n_{3}+n_{4}\right)^{s_{6}}} \\
& \quad \times \frac{(-1)^{l}}{\left(n_{1}+n_{2}\right)^{k}\left(n_{1}+n_{2}-\left(n_{1}+n_{2}+n_{3}+n_{4}\right)\right)^{l}} \\
& =\sum_{j=1}^{k} K_{j} \zeta_{\mathfrak{s l}(5)}\left(s_{1}, 0,0, s_{3}, j, s_{2}, 0, s_{4}, s_{5}, s_{6}+k+l-j\right) \\
& +\sum_{j=1}^{l} L_{j} \zeta_{\mathfrak{s l}(5)}\left(s_{1}, 0,0, s_{3}, 0, s_{2}, j, s_{4}, s_{5}, s_{6}+k+l-j\right) .
\end{aligned}
$$



lemmas.

By the method similar to the proof of Lemma 3.4, we can easily obtain the following

Lemma 3.5. Let $k, l \in \mathbf{N}$. We have

$$
\begin{aligned}
\zeta_{\mathfrak{s l}(3)}(s, k, l)= & \sum_{j=2}^{k}(-1)^{k-j} K_{j} \zeta(j) \zeta(s+k+l-j) \\
& +\sum_{j=2}^{l}(-1)^{k} L_{j} \zeta_{2}(s+k+l-j, j) \\
& +(-1)^{k-1} K_{1}\left(\zeta_{2}(1, s+k+l-1)+\zeta(s+k+l)\right)
\end{aligned}
$$

and

$$
\zeta_{\mathfrak{s l}(3)}(k, l, s)=\sum_{j=1}^{k} K_{j} \zeta_{2}(j, s+k+l-j)+\sum_{j=1}^{l} L_{j} \zeta_{2}(j, s+k+l-j) .
$$

LEMMA 3.6. Let $k, l \in \mathbf{N}$. We have

$$
\begin{aligned}
\zeta_{\mathfrak{s l}(4)}\left(s_{1}, s_{2}, k, s_{3}, 0, l\right)= & \sum_{j=2}^{k}(-1)^{k-j} K_{j} \zeta(j) \zeta_{\mathfrak{s l}(3)}\left(s_{1}, s_{2}, s_{3}+k+l-j\right) \\
& +\sum_{j=2}^{l}(-1)^{k} L_{j} \zeta_{\mathfrak{s l}(4)}\left(s_{1}, s_{2}, 0, s_{3}+k+l-j, 0, j\right) \\
& +(-1)^{k-1} K_{1} \zeta_{\mathfrak{s l}(4)}\left(s_{1}, s_{2}, 1, s_{3}+k+l-2,0,1\right)
\end{aligned}
$$

and

$$
\begin{aligned}
\zeta_{\mathfrak{s l}(4)}\left(k, 0, s_{2}, s_{1}, l, s_{3}\right)= & \sum_{j=1}^{k} K_{j} \zeta_{\mathfrak{s l}(4)}\left(j, 0, s_{2}, s_{1}, 0, s_{3}+k+l-j\right) \\
& +\sum_{j=1}^{l} L_{j} \zeta_{\mathfrak{s l}(4)}\left(0,0, s_{2}, s_{1}, j, s_{3}+k+l-j\right)
\end{aligned}
$$

\section{Proof of main theorems}

In this section we prove Theorem 2.3. By the method similar to the proof of Theorem 2.3, Lemma 3.1, Lemma 3.5 and Lemma 3.6, we can easily obtain Theorem 2.1 and Theorem 2.2 . 
Proof of Theorem 2.3. Let $U\left(s_{1}, s_{2}, s_{3}, s_{4}, s_{5}, s_{6}, k, l\right)$ be the left-hand side of (2.3). Note that we have

$$
\begin{aligned}
& f_{5}\left(s_{1}, s_{2}, s_{3}, 1, s_{4}, s_{5}, 0, s_{6}, 0,1\right)=f_{5}\left(s_{3}, s_{2}, s_{1}, 1, s_{5}, s_{4}, 0, s_{6}, 0,1\right) \\
& =f_{5}\left(s_{3}, s_{2}, 0,0, s_{5}, 0, s_{1}, 1, s_{4}, s_{6}+1\right)+f_{5}\left(s_{3}, 0,0, s_{1}, 1, s_{2}, 0, s_{5}, s_{4}, s_{6}+1\right) \\
& \quad+f_{5}\left(1,0, s_{2}, s_{1}, s_{3}, 0, s_{4}, s_{5}, 0, s_{6}+1\right)+f_{4}\left(s_{3}, s_{2}, s_{1}, s_{5}+1, s_{4}, s_{6}+1\right) \\
& \quad+f_{4}\left(s_{3}+1, s_{2}, s_{1}, s_{5}, s_{4}, s_{6}+1\right)+f_{4}\left(s_{1}, s_{2}, s_{3}, s_{4}, s_{5}, s_{6}+2\right)
\end{aligned}
$$

and

$$
\begin{aligned}
& g(s) f_{4}\left(s_{1}, s_{2}, s_{3}, s_{4}, s_{5}, s_{6}\right)=g(s) f_{4}\left(s_{3}, s_{2}, s_{1}, s_{5}, s_{4}, s_{6}\right) \\
& =f_{5}\left(s_{1}, s_{2}, s_{3}, 0, s_{4}, s_{5}, 0, s_{6}, 0, s\right)+f_{5}\left(s_{3}, s_{2}, 0,0, s_{5}, 0, s_{1}, s, s_{4}, s_{6}\right) \\
& +f_{5}\left(s_{3}, 0,0, s_{1}, s, s_{2}, 0, s_{5}, s_{4}, s_{6}\right)+f_{5}\left(s, 0, s_{2}, s_{1}, s_{3}, 0, s_{4}, s_{5}, 0, s_{6}\right) \\
& +f_{4}\left(s_{1}, s_{2}, s_{3}, s_{4}, s_{5}, s_{6}+s\right)+f_{4}\left(s_{3}, s_{2}, s_{1}, s_{5}+s, s_{4}, s_{6}\right)+f_{4}\left(s_{3}+s, s_{2}, s_{1}, s_{5}, s_{4}, s_{6}\right)
\end{aligned}
$$

by (a), (d), (h) and (h) in Theorem 2.3. By (b), (e), (f), (g) in Theorem 2.3 we have

$$
\begin{aligned}
& U\left(s_{1}, s_{2}, s_{3}, s_{4}, s_{5}, s_{6}, k, l\right) \\
& =\sum_{j=2}^{k}(-1)^{j} K_{j} g(j) f_{4}\left(s_{1}, s_{2}, s_{3}, s_{4}, s_{5}, s_{6}+k+l-j\right) \\
& \quad+\sum_{j=2}^{l} L_{j} f_{5}\left(s_{1}, s_{2}, s_{3}, 0, s_{4}, s_{5}, 0, s_{6}+k+l-j, 0, j\right) \\
& \quad-K_{1} f_{5}\left(s_{1}, s_{2}, s_{3}, 1, s_{4}, s_{5}, 0, s_{6}+k+l-2,0,1\right) \\
& \quad+\sum_{j=2}^{l}(-1)^{j} L_{j} g(j) f_{4}\left(s_{1}, s_{2}, s_{3}, s_{4}, s_{5}, s_{6}+k+l-j\right) \\
& \quad+\sum_{j=2}^{k} K_{j} f_{5}\left(s_{1}, s_{2}, s_{3}, 0, s_{4}, s_{5}, 0, s_{6}+k+l-j, 0, j\right) \\
& \quad-L_{1} f_{5}\left(s_{1}, s_{2}, s_{3}, 1, s_{4}, s_{5}, 0, s_{6}+k+l-2,0,1\right) \\
& \quad+\sum_{j=1}^{k} K_{j} f_{5}\left(j, 0, s_{2}, s_{3}, s_{1}, 0, s_{5}, s_{4}, 0, s_{6}+k+l-j\right) \\
& +\sum_{j=1}^{l} L_{j} f_{5}\left(0,0, s_{2}, s_{3}, s_{1}, 0, s_{5}, s_{4}, j, s_{6}+k+l-j\right)
\end{aligned}
$$




$$
\begin{aligned}
& +\sum_{j=1}^{l} L_{j} f_{5}\left(j, 0, s_{2}, s_{1}, s_{3}, 0, s_{4}, s_{5}, 0, s_{6}+k+l-j\right) \\
& +\sum_{j=1}^{k} K_{j} f_{5}\left(0,0, s_{2}, s_{1}, s_{3}, 0, s_{4}, s_{5}, j, s_{6}+k+l-j\right) \\
& +\sum_{j=1}^{k} K_{j} f_{5}\left(s_{1}, 0,0, s_{3}, j, s_{2}, 0, s_{4}, s_{5}, s_{6}+k+l-j\right) \\
& +\sum_{j=1}^{l} L_{j} f_{5}\left(s_{1}, 0,0, s_{3}, 0, s_{2}, j, s_{4}, s_{5}, s_{6}+k+l-j\right) \\
& =\sum_{j=2}^{k}(-1)^{j} K_{j} g(j) f_{4}\left(s_{1}, s_{2}, s_{3}, s_{4}, s_{5}, s_{6}+k+l-j\right) \\
& +\sum_{j=2}^{l}(-1)^{j} L_{j} g(j) f_{4}\left(s_{1}, s_{2}, s_{3}, s_{4}, s_{5}, s_{6}+k+l-j\right) \\
& +\sum_{j=2}^{k} K_{j}\left(f_{5}\left(s_{1}, s_{2}, s_{3}, 0, s_{4}, s_{5}, 0, s_{6}+k+l-j, 0, j\right)\right. \\
& +f_{5}\left(s_{1}, s_{2}, 0,0, s_{4}, 0, s_{3}, j, s_{5}, s_{6}+k+l-j\right) \\
& +f_{5}\left(s_{1}, 0,0, s_{3}, j, s_{2}, 0, s_{4}, s_{5}, s_{6}+k+l-j\right) \\
& \left.+f_{5}\left(j, 0, s_{2}, s_{3}, s_{1}, 0, s_{5}, s_{4}, 0, s_{6}+k+l-j\right)\right) \\
& +\sum_{j=2}^{l} L_{j}\left(f_{5}\left(s_{1}, s_{2}, s_{3}, 0, s_{4}, s_{5}, 0, s_{6}+k+l-j, 0, j\right)\right. \\
& +f_{5}\left(s_{3}, s_{2}, 0,0, s_{5}, 0, s_{1}, j, s_{4}, s_{6}+k+l-j\right) \\
& +f_{5}\left(s_{3}, 0,0, s_{1}, j, s_{2}, 0, s_{5}, s_{4}, s_{6}+k+l-j\right) \\
& \left.+f_{5}\left(j, 0, s_{2}, s_{1}, s_{3}, 0, s_{4}, s_{5}, 0, s_{6}+k+l-j\right)\right) \\
& +K_{1}\left(-f_{5}\left(s_{1}, s_{2}, s_{3}, 1, s_{4}, s_{5}, 0, s_{6}+k+l-2,0,1\right)\right. \\
& +f_{5}\left(s_{1}, s_{2}, 0,0, s_{4}, 0, s_{3}, 1, s_{5}, s_{6}+k+l-1\right) \\
& +f_{5}\left(s_{1}, 0,0, s_{3}, 1, s_{2}, 0, s_{4}, s_{5}, s_{6}+k+l-1\right) \\
& \left.+f_{5}\left(1,0, s_{2}, s_{3}, s_{1}, 0, s_{5}, s_{4}, 0, s_{6}+k+l-1\right)\right) \\
& +L_{1}\left(-f_{5}\left(s_{1}, s_{2}, s_{3}, 1, s_{4}, s_{5}, 0, s_{6}+k+l-2,0,1\right)\right.
\end{aligned}
$$




$$
\begin{aligned}
& +f_{5}\left(s_{3}, s_{2}, 0,0, s_{5}, 0, s_{1}, 1, s_{4}, s_{6}+k+l-1\right) \\
& +f_{5}\left(s_{3}, 0,0, s_{1}, 1, s_{2}, 0, s_{5}, s_{4}, s_{6}+k+l-1\right) \\
& \left.+f_{5}\left(1,0, s_{2}, s_{1}, s_{3}, 0, s_{4}, s_{5}, 0, s_{6}+k+l-1\right)\right) .
\end{aligned}
$$

By (4.1), (4.2), (d), (h) and (i) we have

$$
\begin{aligned}
& U\left(s_{1}, s_{2}, s_{3}, s_{4}, s_{5}, s_{6}, k, l\right) \\
& =\sum_{j=2}^{k}(-1)^{j} K_{j} g(j) f_{4}\left(s_{1}, s_{2}, s_{3}, s_{4}, s_{5}, s_{6}+k+l-j\right) \\
& +\sum_{j=2}^{l}(-1)^{j} L_{j} g(j) f_{4}\left(s_{1}, s_{2}, s_{3}, s_{4}, s_{5}, s_{6}+k+l-j\right) \\
& +\sum_{j=2}^{k} K_{j}\left(g(j) f_{4}\left(s_{1}, s_{2}, s_{3}, s_{4}, s_{5}, s_{6}+k+l-j\right)\right. \\
& -f_{4}\left(s_{1}, s_{2}, s_{3}, s_{4}, s_{5}, s_{6}+k+l\right)-f_{4}\left(s_{1}, s_{2}, s_{3}, s_{4}+j, s_{5}, s_{6}+k+l-j\right) \\
& \left.-f_{4}\left(s_{1}+j, s_{2}, s_{3}, s_{4}, s_{5}, s_{6}+k+l-j\right)\right) \\
& +\sum_{j=2}^{k} L_{j}\left(g(j) f_{4}\left(s_{1}, s_{2}, s_{3}, s_{4}, s_{5}, s_{6}+k+l-j\right)\right. \\
& -f_{4}\left(s_{1}, s_{2}, s_{3}, s_{4}, s_{5}, s_{6}+k+l\right)-f_{4}\left(s_{3}, s_{2}, s_{1}, s_{5}+j, s_{4}, s_{6}+k+l-j\right) \\
& \left.-f_{4}\left(s_{3}+j, s_{2}, s_{1}, s_{5}, s_{4}, s_{6}+k+l-j\right)\right) \\
& +K_{1}\left(-f_{4}\left(s_{1}, s_{2}, s_{3}, s_{4}, s_{5}, s_{6}+k+l\right)-f_{4}\left(s_{1}, s_{2}, s_{3}, s_{4}+1, s_{5}, s_{6}+k+l-1\right)\right. \\
& \left.-f_{4}\left(s_{1}+1, s_{2}, s_{3}, s_{4}, s_{5}, s_{6}+k+l-1\right)\right) \\
& +L_{1}\left(-f_{4}\left(s_{1}, s_{2}, s_{3}, s_{4}, s_{5}, s_{6}+k+l\right)-f_{4}\left(s_{3}, s_{2}, s_{1}, s_{5}+1, s_{4}, s_{6}+k+l-1\right)\right. \\
& \left.-f_{4}\left(s_{3}+1, s_{2}, s_{1}, s_{5}, s_{4}, s_{6}+k+l-1\right)\right) \\
& =2 \sum_{j=1}^{[k / 2]}\left(\begin{array}{c}
k+l-2 j-1 \\
k-2 j
\end{array}\right) g(2 j) f_{4}\left(s_{1}, s_{2}, s_{3}, s_{4}, s_{5}, s_{6}+k+l-2 j\right) \\
& +2 \sum_{j=1}^{[l / 2]}\left(\begin{array}{c}
k+l-2 j-1 \\
l-2 j
\end{array}\right) g(2 j) f_{4}\left(s_{1}, s_{2}, s_{3}, s_{4}, s_{5}, s_{6}+k+l-2 j\right) \\
& -\sum_{j=1}^{k}\left(\begin{array}{c}
k+l-j-1 \\
k-j
\end{array}\right)\left(f_{4}\left(s_{1}, s_{2}, s_{3}, s_{4}, s_{5}, s_{6}+k+l\right)\right.
\end{aligned}
$$




$$
\begin{aligned}
& \left.+f_{4}\left(s_{1}, s_{2}, s_{3}, s_{4}+j, s_{5}, s_{6}+k+l-j\right)+f_{4}\left(s_{1}+j, s_{2}, s_{3}, s_{4}, s_{5}, s_{6}+k+l-j\right)\right) \\
& -\sum_{j=1}^{l}\left(\begin{array}{c}
k+l-j-1 \\
l-j
\end{array}\right)\left(f_{4}\left(s_{1}, s_{2}, s_{3}, s_{4}, s_{5}, s_{6}+k+l\right)\right. \\
& \left.+f_{4}\left(s_{3}, s_{2}, s_{1}, s_{5}+j, s_{4}, s_{6}+k+l-j\right)+f_{4}\left(s_{3}+j, s_{2}, s_{1}, s_{5}, s_{4}, s_{6}+k+l-j\right)\right) .
\end{aligned}
$$

Since we have

$$
\sum_{j=1}^{k}\left(\begin{array}{c}
k+l-j-1 \\
k-j
\end{array}\right)=\left(\begin{array}{c}
k+l-1 \\
l
\end{array}\right)
$$

by

$$
\left(\begin{array}{c}
k+l-j-1 \\
l-1
\end{array}\right)=\left(\begin{array}{c}
k+l-j-1 \\
k-j
\end{array}\right)
$$

and

$$
\left(\begin{array}{l}
m \\
n
\end{array}\right)=\left(\begin{array}{c}
m-1 \\
n
\end{array}\right)+\left(\begin{array}{c}
m-1 \\
n-1
\end{array}\right),
$$

we obtain (2.3). By Lemma 3.4, we can easily see that we can take $f_{5}=\zeta_{\mathfrak{s l}(5)}, f_{4}=\zeta_{\mathfrak{s l}(4)}$, $g=\zeta$.

\section{References}

[ 1 ] D. M. BRAdley, T. CAI and X. Zhou, Depth reduction of a class of Witten zeta functions, Electron. J. Comb. 16 (2009), N 27.

[ 2 ] M. E. Hoffman, Multiple harmonic series, Pacific J. Math. 152 (1992), 275-290.

[ 3 ] Y. Komori, K. Matsumoto and H. Tsumura, Functional relations for zeta-functions of root systems. in "Number Theory: Dreaming in Dreams", Proc. 5th China-Japan Seminar, T. Aoki et al. (eds.), World Scientific, 2010, pp.135-183.

[ 4 ] Y. Komori, K. Matsumoto and H. Tsumura, On Witten multiple zeta-functions associated with semisimple Lie algebras II, J. Math. Soc. Japan 62 (2010), 355-394.

[ 5 ] K. Matsumoto and H. Tsumura, On Witten multiple zeta-functions associated with semisimple Lie algebras I, Ann. Inst. Fourier 56 (2006), 1457-1504.

[ 6 ] T. Nakamura, A functional relation for the Tornheim double zeta function, Acta Arith. 125 (2006), 257-263.

[ 7 ] T. Nakamura, Double Lerch value relations and functional relations for Witten zeta functions, Tokyo J. Math. 31 (2008), 551-574.

[ 8 ] L. Tornheim, Harmonic double series, Amer. J. Math. 72 (1950), 303-314.

[ 9 ] H. Tsumura, On some combinatorial relations for Tornheim's double series, Acta Arith. 105 (2002), 239252.

[10] H. Tsumura, On functional relations between the Mordell-Tornheim double zeta functions and the Riemann zeta function, Math. Proc. Cambridge Philos. Soc. 142 (2007), 395-405.

[11] E. Witten, On quantum gauge theories in two dimensions, Comm. Math. Phys. 141 (1991), 153-259. 
[12] D. Zagier, Values of zeta functions and their applications, in Proc. First Congress of Math., Paris, vol. II, Progress in Math. 120 (1994), 497-512.

Present Addresses:

SOICHI IKEDA

DEPARTMENT OF MATHEMATICS,

SHIBAURA INSTITUTE OF TECHNOLOGY,

307 FuKASAKU, Minuma-KU, SAITAMa 337-8570, JAPAN.

e-mail: sikeda@sic.shibaura-it.ac.jp

KANEAKI MATSUOKA

GraduATE SCHOOL OF MATHEMATICS,

NAGOYA UNIVERSITY,

Furocho, ChiKus A-KU, NAGOYA 464-8602, JAPAN.

e-mail:m10041v@math.nagoya-u.ac.jp 